\title{
Frequent Errors in Consonant Sound Production of Elementary Education Teachers at Visayas State University
}

\author{
${ }^{1}$ Precious C. Domingo, ${ }^{2}$ Jonathan G. Merillo, and ${ }^{3}$ Mechille Pedrera \\ ${ }^{1}$ Visayas State University, Baybay City \\ ${ }^{2}$ Leyte Normal University, Tacloban City \\ ${ }^{3}$ Casandig National High School, Samar City \\ ${ }^{1}$ precious.domingo@vsu.edu.ph, ${ }^{2}$ jonathan.merillo@1nu.edu.ph, \\ ${ }^{3}$ mechille.pedrera@ deped.gov.ph
}

Article History: Submitted November 12 ${ }^{\text {th }}, 2020$; Accepted December $28^{\text {th }}, 2020$; Published December $30^{\text {th }}, 2020$

\begin{abstract}
Pronunciation is essential in language learning; thus, English learners as a second language should strive for articulateness in sound patterns in English. This study aimed to determine the commonly mispronounced consonant sound of the Bachelor of Elementary Education students of the Visayas State University according to the manner and place of articulation. The instrument used in the study is the Golden-Fristoe Test of Articulation, which is composed of 44 words containing the different consonant sounds in English. The study results revealed that most consonant sounds misarticulated by the respondents were fricatives $/ \mathrm{z} /$, the nasal consonant sound $/ \mathrm{m} /$, the fricative sound $/ ð /$, the affricate $/ \mathrm{dz} /$. With respect to the place of articulation, most consonant sounds misarticulated by the respondents concerning the place of articulation were alveolar $/ \mathrm{z} /$, the velar consonant sound $/ \mathrm{m} /$, the interdental sound $/ ð /$, the post-alveolar /dz/. With these results, it is expected for the Filipino English language teachers to focus on teaching the alveolar and labiodental fricatives in teaching the consonant sounds because most students failed to articulate these sounds correctly.
\end{abstract}

Keywords: English language, consonant sounds, pronunciation

Abstrak. Pelafalan sangat penting dalam pembelajaran bahasa. Oleh karena itu, pelajar bahasa Inggris sebagai bahasa kedua harus berusaha keras untuk mengartikulasikan pola bunyi dalam bahasa Inggris. Penelitian ini bertujuan untuk mengetahui bunyi konsonan yang sering salah diucapkan pada mahasiswa S1 Pendidikan Dasar Universitas Negeri Visayas menurut cara dan tempat artikulasi. Instrumen yang digunakan dalam penelitian ini adalah Golden-Fristoe Test of Articulation, yang terdiri atas 44 kata yang berisi bunyi konsonan yang berbeda dalam bahasa Inggris. Hasil penelitian menunjukkan bahwa bunyi konsonan yang paling banyak salah artikulasi oleh responden adalah bunyi konsonan / z /, bunyi konsonan nasal / $m$ /, bunyi frikatif / $/$ /, afrikat / dz /. Sehubungan dengan tempat artikulasi, sebagian besar bunyi konsonan yang salah diartikulasikan oleh responden mengenai tempat artikulasi adalah alveolar / $z$ /, bunyi konsonan velar / $\mathrm{m} /$, bunyi interdental / $\partial /$, post-alveolar $/ \mathrm{dz} /$. Dengan hasil tersebut diharapkan para pengajar bahasa Inggris Filipina dapat fokus dalam mengajarkan frikatif alveolar dan labiodental dalam mengajarkan bunyi konsonan karena sebagian besar siswa gagal mengucapkan bunyi tersebut dengan benar.

Kata kunci: bahasa Inggris, bunyi konsonan, pelafalan 


\section{INTRODUCTION}

One of the essential skills a person needs in this $21^{\text {st }}$ century is mastery of the English language (Lee, 2009). English emerged as the world's lingua franca. In business agreement and exchange of information, the English language is spoken. Thus using English is deemed as a significant skill in communication. There are around 1.5 billion English language learners worldwide (Knagg, 2019). The desire to speak English with proper pronunciation is vital. Yet, some barriers hinder a speaker from speaking with good English pronunciation. Without achieving a certain degree of accuracy, a speaker can end with an unrecognizable speech (Bazzano, 2016). Many students face difficulties in different language skills. For instance, some students cannot express themselves in English, prompting them to resort to their first language in the classroom. Others feel shy to use English for being afraid of committing errors.

Pronunciation is essential in language learning; thus, learners of English as their second language should aim for articulateness in sound patterns in English, which can help them better understand the meaning of the utterance ( (Wheelock, 2016). Previous studies (Hassan, 2014) stated that errors of non-native speakers of English are systematic and not by accident.

In the Philippine context, the English language is brought to the country by the American soldier. Moreover, for years the English language is used as a second language in the country, even speak at an early age. However, Filipinos still have difficulty in speaking the English language in many aspects, including pronunciation. This study investigates students' common errors in pronouncing words focusing on the consonants commonly misarticulated by Visayas State University students.

\section{Common Errors in Producing Consonant Sounds}

The problems in pronunciation of English are common in many countries that consider English as a second language. These studies show that many non-native English speakers worldwide pronounce both vowels and consonants in English with a variation.

Phonological errors, for example, can be due to the inadequate acquisition of the phonetic/phonemic and phonologic rules of language (Bernthal \& Bankson, 2004). Generally, a child may have a phonological delay if they have not successfully eliminated the phonological processes by a certain age, while children who exhibit unusual processes may have a phonological disorder (Hodson, 2004; Khan, 1982).

In a study conducted by Banu in 2000 in Bangladesh, the researcher observed that Bangladeshi speakers tend to replace unaspirated English consonants with aspirated sounds. The fricative is absent in the Bengali inventory of phonemes. The articulation of a bilabial is aspirated, and stop is standard. The alveolar is somewhat retroflexed. The Bengali rhythm is based on arranging long and short syllables, not stressed, and unstressed ones (Begum \& Azizul Hoque, 2016 ).

In a separate study, Zhang and Yin (2009) analyzed some pronunciation problems often encountered by Chinese English learners. Their observations showed that the factors influencing the pronunciation are first language interference, learner's age, learner's attitude, previous pronunciation instruction received by the learner, the inadequate language of English phonology and phonetics, and differences in words displaying sound-spelling. They observed that the main reason for producing correct English is L1 interference. 
Also, Nuhiu (2012) investigated a range of pronunciation difficulties experienced by original Albanian learners. In a survey, it revealed the problems with the English sounds. Overall, student performance shows that $35 \%$ of students pronounced the back vowels correctly, while the other $65 \%$ pronounced a slight distortion of [o]. In the case of [æ] is pronounced in different variations because of the first language. This sound is pronounced as [e]. Sixtysix percent of the students tested had a successful pronunciation, while $34 \%$ mispronounced it because of the influence of L1 (Nuhiu, 2012) .

In the Philippines, earlier studies have shown some phonological features of American General English (AGE) that are not present in Filipino English (PE), so some Filipino English learners make consonant production mistakes. In the case of consonants, categories that are not present in the Filipino sound system are the labiodental fricatives /f/ (voiceless) and $/ \mathrm{v} /$ (voiced). These are rendered as bilabial stops $/ \mathrm{b} /$ (voiceless) and /b/ (voiced) voiced by Filipino English speakers. However, it is not uniform in the Philippines because/f/ and/v/ are present in specific languages in the Philippines like Ibanag (for example,/bavi/ 'pig', /inafi/ 'boiled rice'). Other examples of consonants present in General America and is absent in Filipino English are the interdental or continuing fricatives $/ \Theta /$ (voiceless) and $/ \delta /$ (voiceless), which are pronounced/t/ and/d/ respectively in PE (Tayao, 2004). Researchers believe that when teaching pronunciation, determining the students' kind of error helps teachers identify and correct this error.

Tayao (2004) suggests in her study that a range of phonemes in Philippine English phonology varies not only in geographical but also in the social aspect. She then concluded that future research agenda on this field should be concerned about one 'standard' Philippine pronunciation and provide descriptions of the different accents due to the geographical and linguistic background and racial-group membership.

A more recent study revealed that using the phonic approach to develop young readers is beneficial. It is more advantageous for young readers to start teaching reading with sounds rather than letters. Learners use their already acquired oral language skills and combine these to sound the written word. The phonemic awareness approach is found to influence teaching reading positively. The below-average readers attain the same amount of knowledge as the high reader. (Gray, et al., 2007)

This study then attempts to identify the common challenges students encounter in producing consonant sounds in the manner and place of articulation.

\section{METHOD}

This study employed a descriptive survey method to help the researchers identify the respondents' common errors in pronouncing consonant sounds according to the place and manner of articulation. This study was conducted among the third-year Bachelor of Elementary Education (BEED) students of Visayas State University. It focused on BEED students since they are expected to teach elementary students where correct sound production is a fundamental phonological skill taught adequately. Using simple random sampling, 30 students were selected among a total of 60 students.

This study used a word list adapted from the validated Golden-Fristoe Test of Articulation (GFTA) instrument created by Dr. Ronald Goldman and Dr. Macalyne Fristoe. The aim of the test is to evaluate the individual articulation of consonants in standard American English. 
The test adopted by the researchers was initially validated in a particular study carried out on 281 Canadian children. The tool was also pilot tested from July to October 1998 and MayNovember 1999.

The Golden-Fristoe Joint Test (GFTA) consists of 44 words that contain various consonant sounds. Respondents read every word on this list. Researchers recorded the activity and later tallied the number of errors committed by the respondents in articulating each word's consonant sounds. The sounds mispronounced by the participants were identified by the researcher based on the manner and place of articulation.

\section{RESULTS AND DISCUSSION}

The study employed a validated instrument Golden-Fristoe Test of Articulation. The words were transcribed to better gather the respondents' errors in producing the consonant sounds, as shown in Table 1.

Table 1 Transcription of the Words from the Instrument

\begin{tabular}{|c|c|}
\hline Word & Transcription \\
\hline house & / 'havs/ \\
\hline telephone & / 'telıfəơn/ \\
\hline cup & / 'kıp/ \\
\hline gun & / 'g $\Lambda \mathrm{n} /$ \\
\hline knife & / 'naIf/ \\
\hline window & / 'windəひ/ \\
\hline wagon & / 'wægən/ \\
\hline wheel & / 'wi:1/ \\
\hline chicken & / 't frkın/ \\
\hline zipper & / 'zipər/ \\
\hline scissors & / 'sIzərz/ \\
\hline duck & / d $\Lambda \mathrm{k} /$ \\
\hline yellow & / jelou/ \\
\hline vacuum & / 'vækjum/ \\
\hline matches & / 'mætfoz/ \\
\hline lamp & / læmp/ \\
\hline shovel & / ' ' $\Lambda$ vəl/ \\
\hline car & / kar/ \\
\hline rabbit & / 'ræbət \\
\hline fishing & / 'fIJIII/ \\
\hline church & / tgarty/ \\
\hline feather & / 'fєðər/ \\
\hline pencils & / 'pensəlz/ \\
\hline This & / ठIS/ \\
\hline Carrot & /'kærət/ \\
\hline Orange & /'orəndz/ \\
\hline bathtub & 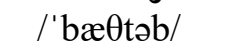 \\
\hline bath & / bæ $\theta$ / \\
\hline thumb & $/ \theta \Lambda \mathrm{m} /$ \\
\hline Finger & / 'fingər/ \\
\hline ring & / rig/ \\
\hline jumping & / 'dz^mpig/ \\
\hline pajamas & / po'dzaməz/ \\
\hline
\end{tabular}




\begin{tabular}{|c|c|}
\hline plane & / plæn/ \\
\hline blue & / blu/ \\
\hline brush & / br $\Lambda \int /$ \\
\hline drum & / dr $\Lambda \mathrm{m} /$ \\
\hline flag & / flæg/ \\
\hline Santa Claus & / 'sæntə kloz/ \\
\hline Christmas tree & / 'krisməs tri/ \\
\hline squirrel & / 'skwərəl/ \\
\hline sleeping & / 'slipıy/ \\
\hline bed & / bed/ \\
\hline stove & / stouv/ \\
\hline
\end{tabular}

\section{Mispronounced Consonant Sounds According to Manner of Articulation}

The first objective of the study was to identify the consonant sound frequently mispronounced by the respondents according to the manner of articulation. The researchers listened to the students' recorded audio as they pronounced each word. Errors committed to pronouncing each word's consonant sounds were tallied. There was a total of 30 students who took the test. The students correctly pronounced the consonant sounds in the following words: cup, gun, knife, window, wagon, wheel, chicken, zipper, duck, yellow, vacuum, lamp, car, rabbit, fishing, church, carrot, bath, finger, ring, jumping, plane, drum, flag, squirrel, sleeping, bed, and stove. On the other hand, the following were the words wherein the consonant sounds were mispronounced by the students: house, telephone, scissors, matches, shovel, feather, pencils, this, orange, bathtub, thumb, pajamas, and Santa Claus.

Table 2 Mispronounced Consonant Sounds According to Manner of Articulation

\begin{tabular}{|c|c|c|c|c|}
\hline Word & Transcription & $\begin{array}{c}\text { No. of students } \\
\text { mispronounce } \\
\text { d the sounds } \\
(\%)\end{array}$ & $\begin{array}{c}\text { No. of students } \\
\text { successfully } \\
\text { pronounced the sounds } \\
(\%)\end{array}$ & $\begin{array}{c}\text { Manner of } \\
\text { Articulation each } \\
\text { Misarticulated Sound } \\
\text { Belongs } \\
\end{array}$ \\
\hline house & / 'havz/ & 0 & $30(100 \%)$ & - \\
\hline$/ \mathrm{h} /$ & & 0 & $30(100 \%)$ & - \\
\hline $\begin{array}{l}\text { /z/ } \\
\text { telephon }\end{array}$ & / 'telıfəon/ & $28(93.33 \%)$ & & Fricative \\
\hline $\mathrm{e}$ & & & & \\
\hline$/ \mathrm{t} /$ & & 0 & $30(100 \%)$ & - \\
\hline$/ 1 /$ & & 0 & $30(100 \%)$ & - \\
\hline /f/ & & $1(3.33 \%)$ & & Fricative \\
\hline$/ \mathrm{n} /$ & & 0 & $30(100 \%)$ & - \\
\hline cup & / 'kıp/ & & & \\
\hline$/ \mathrm{k} /$ & & 0 & $30(100 \%)$ & - \\
\hline$/ \mathrm{p} /$ & & 0 & $30(100 \%)$ & - \\
\hline gun & $/ \operatorname{g} \Lambda \mathrm{n} /$ & & & \\
\hline /g/ & & 0 & $30(100 \%)$ & - \\
\hline$/ \mathrm{n} /$ & & 0 & $30(100 \%)$ & - \\
\hline knife & / 'naif/ & & & \\
\hline$/ \mathrm{n} / \mathrm{kn}$ & & 0 & $30(100 \%)$ & - \\
\hline $\begin{array}{l}\text { /f/ } \\
\text { window }\end{array}$ & / 'windəð/ & 0 & $30(100 \%)$ & - \\
\hline /w/ & & 0 & $30(100 \%)$ & - \\
\hline
\end{tabular}


$/ \mathrm{n} /$

/d/ wagon / 'wægən/

/w/

/g/

/n/

wheel

/w/ wh

/1/

chicken / 't flikın/

$/ \mathrm{t} / \mathrm{ch}$

/k/ ck

$/ \mathrm{n} /$

zipper

/z/

$/ \mathrm{p} / \mathrm{pp}$

$/ \mathrm{r} /$

scissors

/s/ $\mathrm{sc}$

/z/ ss

$/ \mathrm{r} /$

$\mid \mathrm{z} / \mathrm{s}$ duck

/d/

/k/ ck

yellow

/j/ y

/1/ $\quad 11$

vaccum

$/ \mathrm{v} /$

$/ \mathrm{k} / \mathrm{cc}$ $/ \mathrm{m} /$

matches

$/ \mathrm{m} /$

$/ \mathrm{t} /$

$/ \mathrm{t} / \mathrm{ch}$

/z/ s lamp

/1/

$/ \mathrm{m} /$

$/ \mathrm{p} /$

shovel

/'gl sh

$/ \mathrm{v} /$

/l/

car

/c/

/ 'zipər/

/ 'sIzərz/

/ 'jeloo/

/ 'mætfoz/

/ ' 'Avəl/

/ kar/
0

$30(100 \%)$

$30(100 \%)$

$30(100 \%)$

$30(100 \%)$

$30(100 \%)$

$30(100 \%)$

$30(100 \%)$

$30(100 \%)$

$30(100 \%)$

0

$30(100 \%)$

$30(100 \%)$

$30(100 \%)$

0

0

0

$30(100 \%)$

$20(66.67 \%)$

0

$30(100 \%)$

$24(80 \%)$

0

$30(100 \%)$

$30(100 \%)$

0

$30(100 \%)$

$30(100 \%)$

$30(100 \%)$

$30(100 \%)$

$30(100 \%)$

0

$30(100 \%)$

$30(100 \%)$

30 (100\%)

$25(83.33 \%)$

0

$30(100 \%)$

$30(100 \%)$

$30(100 \%)$

0

$2(6.67 \%)$

$1(3.33 \%)$

0

$30(100 \%)$

0

$30(100 \%)$
Fricative

Fricative

Fricative

Fricative

Fricative 


\begin{tabular}{|c|c|c|c|c|}
\hline $\begin{array}{l}\text { /r/ } \\
\text { rabbit }\end{array}$ & / 'ræbət & 0 & $30(100 \%)$ & - \\
\hline$/ \mathrm{r} /$ & & 0 & $30(100 \%)$ & - \\
\hline$/ \mathrm{b} / \quad \mathrm{bb}$ & & 0 & $30(100 \%)$ & - \\
\hline$/ \mathrm{t} /$ & & 0 & $30(100 \%)$ & - \\
\hline fishing & / 'frfiry/ & & & \\
\hline /f/ & & 0 & $30(100 \%)$ & \\
\hline$/ J / \quad$ sh & & 0 & $30(100 \%)$ & - \\
\hline $\begin{array}{l}/ \mathrm{y} / \quad \text { ng } \\
\text { church }\end{array}$ & / tyarty/ & 0 & $30(100 \%)$ & - \\
\hline $\mid \mathrm{tg} / \quad \mathrm{ch}$ & & 0 & $30(100 \%)$ & - \\
\hline$/ \mathrm{r} /$ & & 0 & $30(100 \%)$ & \\
\hline $\begin{array}{l}/ \mathrm{t} / \mathrm{ch} \\
\text { feather }\end{array}$ & / 'fєðər/ & 0 & $30(100 \%)$ & - \\
\hline /f/ & & 0 & $30(100 \%)$ & - \\
\hline$/ ð /$ th & & $9(30 \%)$ & & Fricative \\
\hline $\begin{array}{l}\text { /r/ } \\
\text { pencils }\end{array}$ & / 'pensəlz/ & 0 & $30(100 \%)$ & - \\
\hline$/ \mathrm{p} /$ & & 0 & $30(100 \%)$ & - \\
\hline$/ \mathrm{n} /$ & & 0 & $30(100 \%)$ & - \\
\hline$/ \mathrm{s} / \quad \mathrm{c}$ & & 0 & $30(100 \%)$ & - \\
\hline$/ 1 /$ & & 0 & $30(100 \%)$ & - \\
\hline $\begin{array}{l}\text { /z/ } \quad \mathrm{s} \\
\text { This }\end{array}$ & / ðIS/ & 28 (93.33\%) & & Fricative \\
\hline /ð/ th & & $9(30 \%)$ & & Fricative \\
\hline /s/ & & 0 & $30(100 \%)$ & - \\
\hline Carrot & /'kærət/ & & & \\
\hline$/ \mathrm{k} / \quad \mathrm{c}$ & & 0 & $30(100 \%)$ & - \\
\hline$/ \mathrm{r} / \quad \mathrm{rr}$ & & 0 & $30(100 \%)$ & - \\
\hline$/ \mathrm{t} /$ & & 0 & $30(100 \%)$ & - \\
\hline Orange & /'orondz/ & & & \\
\hline$/ \mathrm{r} /$ & & 0 & $30(100 \%)$ & - \\
\hline$/ \mathrm{n} /$ & & $1(3.33 \%)$ & & Fricative \\
\hline $\begin{array}{l}\text { / d } / \mathrm{g} \\
\text { bathtub }\end{array}$ & /'bæ0tab/ & 0 & $30(100 \%)$ & - \\
\hline$/ \mathrm{b} /$ & & 0 & $30(100 \%)$ & - \\
\hline$/ \theta /$ th & & $3(10 \%)$ & & Fricative \\
\hline$/ \mathrm{t} /$ & & 0 & $30(100 \%)$ & - \\
\hline $\begin{array}{l}/ \mathrm{b} / \\
\text { bath }\end{array}$ & / bæ $\theta /$ & 0 & $30(100 \%)$ & - \\
\hline$/ \mathrm{b} /$ & & 0 & $30(100 \%)$ & - \\
\hline $\begin{array}{l}/ \theta / \text { th } \\
\text { thumb }\end{array}$ & / $\theta \Lambda \mathrm{m} /$ & 0 & $30(100 \%)$ & - \\
\hline$/ \theta /$ th & & 0 & $30(100 \%)$ & - \\
\hline $\begin{array}{l}/ \mathrm{m} / \\
\mathrm{mb}\end{array}$ & & 28 (93.33\%) & & Nasal \\
\hline Finger & / 'frngər/ & & & \\
\hline /f/ & & 0 & $30(100 \%)$ & - \\
\hline
\end{tabular}




\begin{tabular}{|c|c|c|c|c|}
\hline$/ \mathrm{y} /$ & & 0 & $30(100 \%)$ & - \\
\hline$/ g /$ & & 0 & $30(100 \%)$ & - \\
\hline$/ \mathrm{r} /$ & & 0 & $30(100 \%)$ & - \\
\hline ring & / rin/ & & & \\
\hline$/ \mathrm{r} /$ & & 0 & $30(100 \%)$ & - \\
\hline $\begin{array}{l}/ \mathrm{y} / \quad \text { ng } \\
\text { jumping }\end{array}$ & / 'dz^mpin/ & 0 & $30(100 \%)$ & - \\
\hline /dz/ & & 0 & $30(100 \%)$ & - \\
\hline$/ \mathrm{m} /$ & & 0 & $30(100 \%)$ & - \\
\hline$/ \mathrm{p} /$ & & 0 & $30(100 \%)$ & - \\
\hline /ng/ & & 0 & $30(100 \%)$ & - \\
\hline pajamas & / pə'dzaməz/ & & & \\
\hline$/ \mathrm{p} /$ & & 0 & $30(100 \%)$ & - \\
\hline$/ \mathrm{d} / \mathrm{j}$ & & $12(40 \%)$ & & Affricate \\
\hline$/ \mathrm{m} /$ & & 0 & $30(100 \%)$ & - \\
\hline $\begin{array}{l}\mid \mathrm{z} / \\
\text { plane }\end{array}$ & / plæn/ & $25(83.33 \%)$ & & Fricative \\
\hline$/ \mathrm{p} /$ & & 0 & $30(100 \%)$ & - \\
\hline$/ 1 /$ & & 0 & $30(100 \%)$ & - \\
\hline /n/ & / blu/ & 0 & $30(100 \%)$ & - \\
\hline$/ \mathrm{b} /$ & & 0 & $30(100 \%)$ & - \\
\hline $\begin{array}{l}\text { /1/ } \\
\text { brush }\end{array}$ & / br $\Lambda \int /$ & 0 & $30(100 \%)$ & - \\
\hline$/ \mathrm{b} /$ & & 0 & $30(100 \%)$ & - \\
\hline$/ \mathrm{r} /$ & & $1(3.33 \%)$ & & Approximant \\
\hline $\begin{array}{c}/ \int / \text { sh } \\
\text { drum }\end{array}$ & $/ \mathrm{dr} \Lambda \mathrm{m} /$ & 0 & $30(100 \%)$ & - \\
\hline$/ \mathrm{d} /$ & & 0 & $30(100 \%)$ & - \\
\hline$/ \mathrm{r} /$ & & 0 & $30(100 \%)$ & - \\
\hline $\begin{array}{l}/ \mathrm{m} / \\
\quad \text { flag }\end{array}$ & / flæg/ & 0 & $30(100 \%)$ & - \\
\hline /f/ & & 0 & $30(100 \%)$ & - \\
\hline$/ 1 /$ & & 0 & $30(100 \%)$ & - \\
\hline$/ g /$ & & 0 & $30(100 \%)$ & - \\
\hline $\begin{array}{l}\text { Santa } \\
\text { Claus }\end{array}$ & / 'sæntə kləz/ & & & \\
\hline /S/ & & 0 & $30(100 \%)$ & \\
\hline$/ \mathrm{n} /$ & & 0 & $30(100 \%)$ & - \\
\hline$/ \mathrm{t} /$ & & 0 & $30(100 \%)$ & - \\
\hline$/ \mathrm{k} / \quad \mathrm{c}$ & & 0 & $30(100 \%)$ & - \\
\hline$/ 1 /$ & & 0 & $30(100 \%)$ & - \\
\hline /z/ s & & $28(93.33 \%)$ & & Fricative \\
\hline $\begin{array}{l}\text { Christm } \\
\text { as tree }\end{array}$ & / 'krismos tri/ & & & \\
\hline$/ \mathrm{k} / \quad \mathrm{ch}$ & & 0 & $30(100 \%)$ & - \\
\hline$/ \mathrm{r} /$ & & 0 & $30(100 \%)$ & - \\
\hline /s/ st & & $8(26.67 \%)$ & & Fricative \\
\hline
\end{tabular}




\begin{tabular}{|c|c|c|c|c|}
\hline$/ \mathrm{m} /$ & & 0 & $30(100 \%)$ & - \\
\hline /s/ & & 0 & $30(100 \%)$ & - \\
\hline$/ \mathrm{tr} /$ & & 0 & $30(100 \%)$ & - \\
\hline squirrel & / 'skwərəl/ & & & \\
\hline$/ \mathrm{s} /$ & & 0 & $30(100 \%)$ & - \\
\hline$/ \mathrm{kw} / \mathrm{q}$ & & 0 & $30(100 \%)$ & - \\
\hline$/ \mathrm{r} / \quad \mathrm{rr}$ & & 0 & $30(100 \%)$ & - \\
\hline /1/ & & 0 & $30(100 \%)$ & - \\
\hline sleeping & / 'slipın/ & & & \\
\hline /s/ & & 0 & $30(100 \%)$ & - \\
\hline /1/ & & 0 & $30(100 \%)$ & - \\
\hline$/ \mathrm{p} /$ & & 0 & $30(100 \%)$ & - \\
\hline$/ \mathrm{y} / \quad \mathrm{ng}$ & & 0 & $30(100 \%)$ & - \\
\hline bed & / bed/ & & & \\
\hline$/ \mathrm{b} /$ & & 0 & $30(100 \%)$ & - \\
\hline$/ \mathrm{d} /$ & & 0 & $30(100 \%)$ & - \\
\hline stove & / storv/ & & & \\
\hline /s/ & & 0 & $30(100 \%)$ & - \\
\hline$/ t /$ & & 0 & $30(100 \%)$ & - \\
\hline$/ \mathrm{v} /$ & & 0 & $30(100 \%)$ & - \\
\hline
\end{tabular}

The consonant sounds misarticulated by the respondents are fricatives $/ z /$ in the word 'house.' Most respondents substituted /z/ with /s/. The fricative /z/ was also mispronounced in the words 'matches', 'pencils' 'pajamas', and 'Santa Claus.' As a rule, the letter "s" is pronounced as /z/ when it follows a voiced consonant such as (b,d,g,j,l,m,n). Vowel letters and diphthongs are all voiced sounds; thus, if followed with the letter "s," it is pronounced as "z" or "iz" like in the word pajamas and Santa Claus.

The respondents mispronounced the nasal consonant sound $/ \mathrm{m} /$ in the word 'thumb,' producing $/ \mathrm{mb} /$ instead of $/ \mathrm{m} /$ where $/ \mathrm{b} /$ in 'thumb' is silenced. Thumb has two consonant sounds, the consonant cluster th was pronounced as $/ \theta /$ as in theta, and the letters mb should be pronounced as $/ \mathrm{m} /$ with silent $\mathrm{b}$. The error was manifested in articulating the final sound, where respondents pronounced the word with the last $/ \mathrm{mb} /$ sound instead of silent $b$. Students also mispronounced the fricative sound / $/ /$ in words 'feather' and 'this,' the affricate /dz/ in 'pajamas,' the fricative consonant /s/ where respondents pronounced the consonant $/ \mathrm{s} /$ in 'Christmas tree' as /st/ not silencing the consonant $/ \mathrm{t} /$.

Other consonant sounds mispronounced by the respondents are: $/ \Theta /$ in bathtub, $/ \delta /$ in 'shovel', /f/ in telephone, /v/ in shovel, /n/ in orange, and approximant /r/ in brush.

\section{Mispronounced Consonant Sounds According to Place of Articulation}

The second objective of the study was to identify the consonant sound frequently mispronounced by the respondents according to the place of articulation. Errors committed to pronouncing each word's consonant sounds were tallied while the researchers listened to the recorded audio of the 30 students' respondents. The students correctly pronounced the consonant sounds in the following words: cup, gun, knife, window, wagon, wheel, chicken, zipper, duck, yellow, vacuum, lamp, car, rabbit, fishing, church, carrot, bath, finger, ring, jumping, plane, drum, flag, squirrel, sleeping, bed, and stove. On the other hand, the following were the words wherein the consonant sounds were mispronounced by the 
students: house, telephone, scissors, matches, shovel, feather, pencils, this, orange, bathtub, thumb, pajamas, and Santa Claus.

Table 3 Mispronounced Consonant Sounds According to Manner of Articulation

\begin{tabular}{|c|c|c|c|c|}
\hline Word & Transcription & $\begin{array}{l}\text { No. of students } \\
\text { mispronounced the } \\
\text { sounds } \\
(\%)\end{array}$ & $\begin{array}{l}\text { No. of students } \\
\text { successfully } \\
\text { pronounced the } \\
\text { sounds }(\%)\end{array}$ & $\begin{array}{c}\text { Manner of } \\
\text { Articulation each } \\
\text { Misarticulated } \\
\text { Sound Belongs } \\
\end{array}$ \\
\hline house & / 'havz/ & 0 & $30(100 \%)$ & - \\
\hline$/ \mathrm{h} /$ & & 0 & $30(100 \%)$ & - \\
\hline |z/ & & $28(93.33 \%)$ & & Alveolar \\
\hline telephone & / 'telıfəon/ & & & \\
\hline$/ \mathrm{t} /$ & & 0 & $30(100 \%)$ & - \\
\hline$/ 1 /$ & & 0 & $30(100 \%)$ & - \\
\hline /f/ & & $1(3.33 \%)$ & & Labiodental \\
\hline$/ \mathrm{n} /$ & & 0 & $30(100 \%)$ & - \\
\hline cup & / 'kıp/ & & & \\
\hline$/ \mathrm{k} /$ & & 0 & $30(100 \%)$ & - \\
\hline$/ \mathrm{p} /$ & & 0 & $30(100 \%)$ & - \\
\hline gun & $/ \operatorname{g} \Lambda \mathrm{n} /$ & & & \\
\hline /g/ & & 0 & $30(100 \%)$ & - \\
\hline$/ \mathrm{n} /$ & & 0 & $30(100 \%)$ & - \\
\hline knife & / 'naif/ & & & \\
\hline$/ \mathrm{n} / \quad \mathrm{kn}$ & & 0 & $30(100 \%)$ & - \\
\hline /f/ & & 0 & $30(100 \%)$ & - \\
\hline window & / 'windəə// & & & \\
\hline /w/ & & 0 & $30(100 \%)$ & - \\
\hline$/ \mathrm{n} /$ & & 0 & $30(100 \%)$ & - \\
\hline$/ \mathrm{d} /$ & & 0 & $30(100 \%)$ & \\
\hline wagon & / 'wægən/ & & & - \\
\hline /w/ & & 0 & $30(100 \%)$ & - \\
\hline /g/ & & 0 & $30(100 \%)$ & - \\
\hline$/ \mathrm{n} /$ & & 0 & $30(100 \%)$ & \\
\hline wheel & / 'wi:1/ & & & - \\
\hline /w/ wh & & 0 & $30(100 \%)$ & - \\
\hline /1/ & & 0 & $30(100 \%)$ & - \\
\hline chicken & / 't $\int \mathrm{IkIn} /$ & & & \\
\hline$/ \mathrm{t} / \quad \mathrm{ch}$ & & 0 & $30(100 \%)$ & - \\
\hline$/ \mathrm{k} / \quad \mathrm{ck}$ & & 0 & $30(100 \%)$ & - \\
\hline$/ \mathrm{n} /$ & & 0 & $30(100 \%)$ & \\
\hline zipper & / 'zipər/ & & & \\
\hline$|z|$ & & 0 & $30(100 \%)$ & - \\
\hline$/ \mathrm{p} / \quad \mathrm{pp}$ & & 0 & $30(100 \%)$ & - \\
\hline$/ \mathrm{r} /$ & & 0 & $30(100 \%)$ & - \\
\hline
\end{tabular}




\begin{tabular}{|c|c|c|c|c|}
\hline /s/ $\quad \mathrm{sc}$ & & 0 & $30(100 \%)$ & - \\
\hline /z/ ss & & $20(66.67 \%)$ & & Alveolar \\
\hline$/ \mathrm{r} /$ & & 0 & $30(100 \%)$ & - \\
\hline /z/ $\quad \mathrm{s}$ & & $24(80 \%)$ & & Alveolar \\
\hline duck & $/ \mathrm{d} \Lambda \mathrm{k} /$ & & & \\
\hline$/ \mathrm{d} /$ & & 0 & $30(100 \%)$ & - \\
\hline $\begin{array}{l}\text { /k/ ck } \\
\text { yellow }\end{array}$ & / 'jelow/ & 0 & $30(100 \%)$ & - \\
\hline$/ \mathrm{j} / \quad \mathrm{y}$ & & 0 & $30(100 \%)$ & - \\
\hline$/ 1 / \quad 11$ & & 0 & $30(100 \%)$ & - \\
\hline vaccum & / 'vækjum/ & & & \\
\hline$/ \mathrm{v} /$ & & 0 & $30(100 \%)$ & - \\
\hline$/ \mathrm{k} / \quad \mathrm{cc}$ & & 0 & $30(100 \%)$ & - \\
\hline$/ \mathrm{m} /$ & & 0 & $30(100 \%)$ & - \\
\hline matches & / 'mætfoz/ & & & \\
\hline$/ \mathrm{m} /$ & & 0 & $30(100 \%)$ & - \\
\hline$/ \mathrm{t} /$ & & 0 & $30(100 \%)$ & - \\
\hline$/ \mathrm{t} / \mathrm{ch}$ & & 0 & $30(100 \%)$ & - \\
\hline $\mid z / \quad \mathrm{s}$ & & $25(83.33 \%)$ & & Alveolar \\
\hline lamp & / læmp/ & & & \\
\hline$/ 1 /$ & & 0 & $30(100 \%)$ & - \\
\hline$/ \mathrm{m} /$ & & 0 & $30(100 \%)$ & - \\
\hline$/ \mathrm{p} /$ & & 0 & $30(100 \%)$ & - \\
\hline shovel & 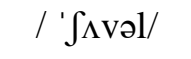 & & & \\
\hline$/$ 'Jl sh & & $2(6.67 \%)$ & & Post-Alveolar \\
\hline$/ \mathrm{v} /$ & & $1(3.33 \%)$ & & Labiodental \\
\hline$/ 1 /$ & & 0 & $30(100 \%)$ & \\
\hline car & / kar/ & & & \\
\hline /c/ & & 0 & $30(100 \%)$ & \\
\hline$/ \mathrm{r} /$ & & 0 & $30(100 \%)$ & - \\
\hline rabbit & / 'ræbət & & & \\
\hline$/ \mathrm{r} /$ & & 0 & $30(100 \%)$ & - \\
\hline$/ \mathrm{b} / \quad \mathrm{bb}$ & & 0 & $30(100 \%)$ & - \\
\hline$/ \mathrm{t} /$ & & 0 & $30(100 \%)$ & - \\
\hline fishing & / 'frf $\mathrm{In} /$ & & & \\
\hline /f/ & & 0 & $30(100 \%)$ & \\
\hline IS/ sh & & 0 & $30(100 \%)$ & - \\
\hline$/ \mathrm{y} / \quad \mathrm{ng}$ & & 0 & $30(100 \%)$ & - \\
\hline church & / tyartg/ & & & \\
\hline$/ \mathrm{t} / \quad \mathrm{ch}$ & & 0 & $30(100 \%)$ & - \\
\hline$/ \mathrm{r} /$ & & 0 & $30(100 \%)$ & \\
\hline $\begin{array}{l}\mid \mathrm{f} / \mathrm{ch} \\
\text { feather }\end{array}$ & / 'fєðər/ & 0 & $30(100 \%)$ & - \\
\hline /f/ & & 0 & $30(100 \%)$ & - \\
\hline$/ ð /$ th & & $9(30 \%)$ & & Interdental \\
\hline
\end{tabular}




\begin{tabular}{|c|c|c|c|c|}
\hline $\begin{array}{l}/ \mathrm{r} / \\
\quad \text { pencils }\end{array}$ & / 'pensəlz/ & 0 & $30(100 \%)$ & - \\
\hline$/ \mathrm{p} /$ & & 0 & $30(100 \%)$ & - \\
\hline$/ \mathrm{n} /$ & & 0 & $30(100 \%)$ & - \\
\hline$/ \mathrm{s} / \quad \mathrm{c}$ & & 0 & $30(100 \%)$ & - \\
\hline /1/ & & 0 & $30(100 \%)$ & - \\
\hline $\mid \mathrm{z} / \quad \mathrm{s}$ & & 28 (93.33\%) & & Alveolar \\
\hline This & / ðIS/ & & & \\
\hline /ð / th & & $9(30 \%)$ & & Interdental \\
\hline /s/ & & 0 & $30(100 \%)$ & - \\
\hline Carrot & /'kærət/ & & & \\
\hline$/ \mathrm{k} / \quad \mathrm{c}$ & & 0 & $30(100 \%)$ & - \\
\hline$/ \mathrm{r} / \quad \mathrm{rr}$ & & 0 & $30(100 \%)$ & - \\
\hline$/ \mathrm{t} /$ & & 0 & $30(100 \%)$ & - \\
\hline Orange & /'orəndz/ & & & \\
\hline$/ \mathrm{r} /$ & & 0 & $30(100 \%)$ & - \\
\hline$/ \mathrm{n} /$ & & $1(3.33 \%)$ & & Velar \\
\hline $\begin{array}{l}\text { / ds/ g } \\
\text { bathtub }\end{array}$ & /'bæ $\theta t ə b /$ & 0 & $30(100 \%)$ & \\
\hline /b/ & & 0 & $30(100 \%)$ & - \\
\hline$/ \theta / \quad$ th & & $3(10 \%)$ & & Interdental \\
\hline$/ \mathrm{t} /$ & & 0 & $30(100 \%)$ & - \\
\hline /b/ & & 0 & $30(100 \%)$ & - \\
\hline bath & / bæ $\theta /$ & & & \\
\hline /b/ & & 0 & $30(100 \%)$ & - \\
\hline $\begin{array}{l}/ \theta / \text { th } \\
\text { thumb }\end{array}$ & $/ \theta \Lambda \mathrm{m} /$ & 0 & $30(100 \%)$ & - \\
\hline$/ \theta / \quad$ th & & 0 & $30(100 \%)$ & - \\
\hline$/ \mathrm{m} / \quad \mathrm{mb}$ & & $28(93.33 \%)$ & & Velar \\
\hline Finger & / 'fingər/ & & & \\
\hline /f/ & & 0 & $30(100 \%)$ & - \\
\hline$/ \mathrm{y} /$ & & 0 & $30(100 \%)$ & - \\
\hline /g/ & & 0 & $30(100 \%)$ & - \\
\hline$/ \mathrm{r} /$ & & 0 & $30(100 \%)$ & - \\
\hline ring & / rin/ & & & \\
\hline$/ \mathrm{r} /$ & & 0 & $30(100 \%)$ & - \\
\hline $\begin{array}{l}/ \mathrm{y} / \quad \mathrm{ng} \\
\text { jumping }\end{array}$ & / 'dsımpig/ & 0 & $30(100 \%)$ & - \\
\hline$/ \mathrm{dz} /$ & & 0 & $30(100 \%)$ & - \\
\hline$/ \mathrm{m} /$ & & 0 & $30(100 \%)$ & - \\
\hline$/ \mathrm{p} /$ & & 0 & $30(100 \%)$ & - \\
\hline /ng/ & & 0 & $30(100 \%)$ & - \\
\hline pajamas & / pə'dzaməz/ & & & \\
\hline$/ \mathrm{p} /$ & & 0 & $30(100 \%)$ & - \\
\hline
\end{tabular}




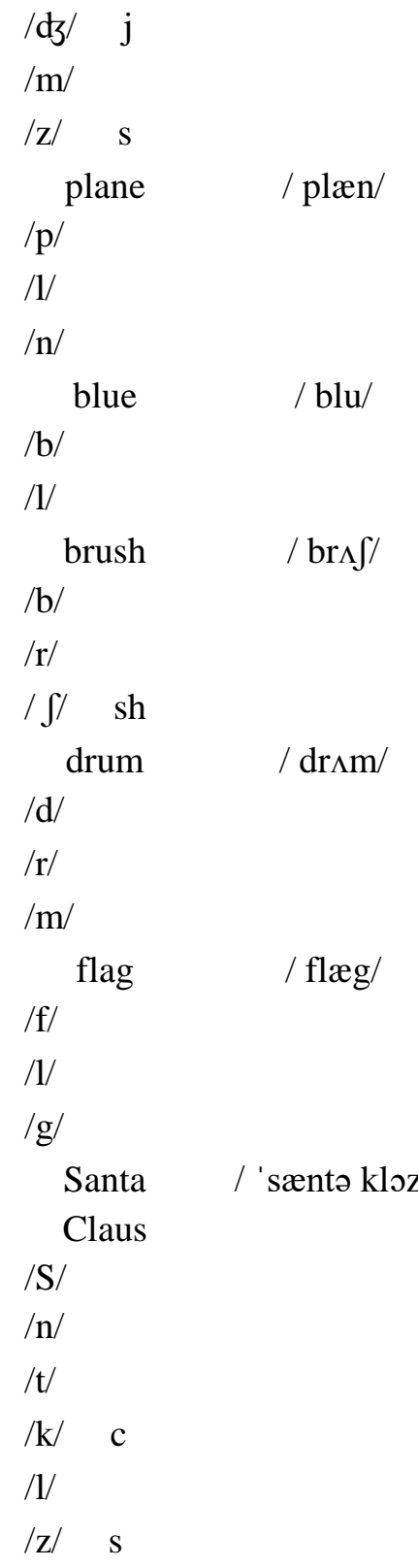

Christmas / 'krisməs tri/ tree

/k/ ch

/r/

/s/ st

$/ \mathrm{m} /$

/s/

/tr/

squirrel / 'skwərəl/

/s/

$/ \mathrm{kw} / \mathrm{q}$

/r/ rr

/1/

sleeping / / slipı/
$12(40 \%)$

0

$25(83.33 \%)$

$30(100 \%)$

$\begin{array}{ll}0 & 30(100 \%) \\ 0 & 30(100 \%) \\ 0 & 30(100 \%)\end{array}$

$30(100 \%)$

$30(100 \%)$

$30(100 \%)$

$1(3.33 \%)$

0

$30(100 \%)$

$30(100 \%)$

$30(100 \%)$

30 (100\%)

$30(100 \%)$

$30(100 \%)$

30 (100\%)

$30(100 \%)$

30 (100\%)

$30(100 \%)$

$30(100 \%)$

$30(100 \%)$

$28(93.33 \%)$

Labiodental

$30(100 \%)$

$30(100 \%)$

$8(26.67 \%)$

$30(100 \%)$

$30(100 \%)$

$30(100 \%)$

0

0

$30(100 \%)$

$30(100 \%)$

$30(100 \%)$

$30(100 \%)$

Post-alveolar

Labiodental

Alveolar
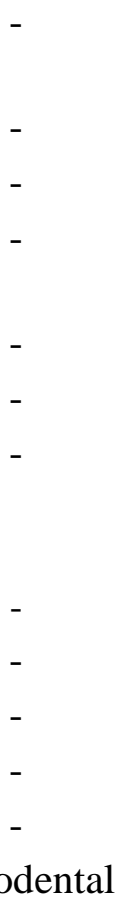

Alveolar

0

0

$\begin{array}{ll}0 & 30(100 \%) \\ 0 & 30(100 \%) \\ 0 & 30(100 \%)\end{array}$




\begin{tabular}{|c|c|c|c|c|}
\hline$/ \mathrm{s} /$ & & 0 & $30(100 \%)$ & - \\
\hline$/ 1 /$ & & 0 & $30(100 \%)$ & - \\
\hline$/ \mathrm{p} /$ & & 0 & $30(100 \%)$ & - \\
\hline$/ \mathrm{y} / \quad \mathrm{ng}$ & & 0 & $30(100 \%)$ & - \\
\hline bed & $/ \mathrm{b} \varepsilon \mathrm{d} /$ & & & \\
\hline$/ \mathrm{b} /$ & & 0 & $30(100 \%)$ & - \\
\hline$/ \mathrm{d} /$ & & 0 & $30(100 \%)$ & - \\
\hline stove & / stouv/ & & & \\
\hline /s/ & & 0 & $30(100 \%)$ & - \\
\hline$/ \mathrm{t} /$ & & 0 & $30(100 \%)$ & - \\
\hline$/ \mathrm{v} /$ & & 0 & $30(100 \%)$ & - \\
\hline
\end{tabular}

Most consonant sounds misarticulated by the respondents concerning the place of articulation are alveolar /z/, where most respondents substituted /z/ with /s/. The alveolar /z/ was also mispronounced in the words 'matches' with $83.33 \%$ students, 'pencils' with 93.33\%, 'pajamas' with $83.33 \%$, and 'Santa Claus' with $93.33 \%$.

The velar consonant sound $/ \mathrm{m} /$ in the word 'thumb' was mispronounced by $93.33 \%$ of the respondents. Thirty percent of the students mispronounced the interdental sound / $/$ / in the words 'feather' and 'this.'

The post-alveolar $/ \mathrm{dz} /$ in 'pajamas' was mispronounced by $40 \%$ of the respondents. The alveolar consonant /s/ is also mispronounced $26.67 \%$ of the respondents. Other consonant sounds mispronounced by the respondents are: interdental $/ \Theta /$ in bathtub with $10 \%$ respondents; post alveolar $/ \mathrm{sh} /$ in 'shovel' with $6.67 \%$; labiodental /f/ in telephone with $3.33 \%$; labiodental /v/ in shovel with $3.33 \%$; velar $/ \mathrm{n} /$ in orange with $3.33 \%$, and alveolar $/ \mathrm{r} /$ in brush with $3.33 \%$.

Table 4 Total Consonant Sound Error in Each Word

\begin{tabular}{cc}
\hline Word & Total number of Error \\
\hline House & 28 \\
Telephone & 1 \\
Cup & 0 \\
Gun & 0 \\
Knife & 0 \\
Window & 0 \\
Wagon & 0 \\
Wheel & 0 \\
Chicken & 0 \\
Zipper & 0 \\
Scissors & 44 \\
Duck & 0 \\
Yellow & 0 \\
Vacuum & 0 \\
Matches & 25 \\
Lamp & 0 \\
Shovel & 1 \\
Car & 0 \\
Rabbit & 0 \\
Fishing & 0 \\
\hline
\end{tabular}




\begin{tabular}{cc}
\hline Church & 0 \\
Feather & 0 \\
Pencils & 28 \\
This & 9 \\
Carrot & 0 \\
Orange & 1 \\
Bathtub & 3 \\
Bath & 0 \\
Thumb & 28 \\
Finger & 0 \\
Ring & 0 \\
Jumping & 0 \\
Pajamas & 25 \\
Plane & 0 \\
Blue & 0 \\
Brush & 0 \\
Drum & 0 \\
Flag & 0 \\
Santa Claus & 28 \\
Christmas tree & 8 \\
Squirrel & 0 \\
Sleeping & 0 \\
Bed & 0 \\
Stove & 0 \\
\hline
\end{tabular}

The words that were mispronounced were tallied. The most mispronounced word was scissors with a total of 44 errors, followed by the words house, pencils, thumb, Santa Claus, each with 28 errors. The word matches and pajamas had 25 errors each, jumping had 12 errors, while the pronoun this had nine errors. The word Christmas tree and bathtub had 8 and 3 errors while the words telephone and shovel had one error each.

Scissors /'sizərz/ has four consonant sounds. The letters sc was pronounced as /s/, ss was pronounced as $/ \mathrm{z} /, \mathrm{r}$ was pronounced as/r/, and the final letter $\mathrm{s}$ was pronounced as $/ \mathrm{z} /$. The error in pronouncing the word commonly occurred in pronouncing ss as /z/ and in the final letter s. Instead of pronouncing the letter s as /z/, respondents pronounced it as /s/. The same was true in the word house. The consonant letter in the word where the letter $h$ is pronounced as $/ \mathrm{h} /$ and the letter s pronounced as $/ \mathrm{z} /$. The error occurred on the second consonant sound, where students pronounce the end sound /s/ instead of /z/. For the word pencils, an error occurred on the final sound, where respondents pronounced it as /s/ instead of /z/. Thumb has two consonant sounds, the consonant cluster th was pronounced as $/ \theta /$ as in theta, and the letters mb should be pronounced as $/ \mathrm{m} /$ with silent $\mathrm{b}$. The error was manifested in articulating the final sound, where respondents pronounced the word with the final $/ \mathrm{mb} /$ sound instead of silent b. For the word Santa Claus, matches, and pajamas, an error was still in pronouncing the word's final sound. Respondents pronounce the final sound as $/ \mathrm{s} /$ instead of /z/. For the word, this, the error was in the initial sound. Instead of making the sound /ð/, respondents articulated the sound $/ \mathrm{d} /$. For the word Christmas tree, an error occurred in pronouncing the word Christmas. Instead of reading the word with silent $t$, respondents included the sound /t/ in reading the word. For the word bathtub, the error occurred in articulating the word bath; respondents pronounced it as /bat/. For telephone, one respondent pronounced the word phone with the ph sound /p/, and for the shovel, pronounced $/ \mathrm{v} /$ as $/ \mathrm{b} /$. 
Table 5 Summary of the Errors in Pronouncing the Consonant Sounds

\begin{tabular}{|c|c|c|c|}
\hline $\begin{array}{l}\text { Consonant } \\
\text { letter }\end{array}$ & $\begin{array}{l}\text { Consonant } \\
\text { Sound }\end{array}$ & $\begin{array}{l}\text { No of times the sound } \\
\text { appeared in the } \\
\text { instrument }\end{array}$ & $\begin{array}{l}\text { Total no of errors in } \\
\text { pronouncing the sound }\end{array}$ \\
\hline $\mathrm{h}$ & $/ \mathrm{h} /$ & 1 & 0 \\
\hline $\mathrm{s}$ & $/ \mathrm{s} /$ & 9 & 0 \\
\hline $\mathrm{t}$ & $/ \mathrm{t} /$ & 8 & 8 \\
\hline 1 & $/ 1 /$ & 11 & 0 \\
\hline $\mathrm{f}$ & /f/ & 6 & 0 \\
\hline $\mathrm{n}$ & $/ \mathrm{n} /$ & 8 & 0 \\
\hline $\mathrm{k}$ & $/ \mathrm{k} /$ & 1 & 0 \\
\hline $\mathrm{p}$ & $/ \mathrm{p} /$ & 8 & 0 \\
\hline $\mathrm{g}$ & /g/ & 4 & 0 \\
\hline $\mathrm{w}$ & $/ \mathrm{w} /$ & 2 & 0 \\
\hline $\mathrm{z}$ & $|z|$ & 1 & 0 \\
\hline $\mathrm{r}$ & $/ \mathrm{r} /$ & 14 & 0 \\
\hline $\mathrm{d}$ & $/ \mathrm{d} /$ & 3 & 0 \\
\hline $\mathrm{m}$ & $/ \mathrm{m} /$ & 7 & 0 \\
\hline $\mathrm{y}$ & /j/ & 1 & 0 \\
\hline $\mathrm{v}$ & $/ \mathrm{v} /$ & 3 & 1 \\
\hline $\mathrm{b}$ & $/ \mathrm{b} /$ & 7 & 0 \\
\hline $\mathrm{j}$ & $/ \mathrm{dz} /$ & 3 & 0 \\
\hline $\mathrm{kn}$ & $/ \mathrm{n} /$ & 1 & 0 \\
\hline wh & $/ \mathrm{w} /$ & 1 & 0 \\
\hline $\mathrm{ch}$ & $\mid \mathrm{tg} /$ & 4 & 0 \\
\hline $\mathrm{ch}$ & $/ \mathrm{k} /$ & 1 & 0 \\
\hline $\mathrm{ck}$ & $/ \mathrm{k} /$ & 2 & 0 \\
\hline sh & IJI & 3 & 0 \\
\hline $\mathrm{s}, \mathrm{ss}$ & $|z|$ & 7 & 178 \\
\hline th & /ð/ & 2 & 9 \\
\hline th & $/ \theta /$ & 3 & 3 \\
\hline $\mathrm{c}$ & $/ \mathrm{k} /$ & 3 & 0 \\
\hline $\mathrm{cl}$ & $/ \mathrm{kl} /$ & 1 & 0 \\
\hline $\mathrm{mb}$ & $/ \mathrm{m} /$ & 1 & 28 \\
\hline $\mathrm{ng}$ & $/ \mathrm{y}$ & 5 & 0 \\
\hline $\mathrm{ph}$ & $/ \mathrm{f} /$ & 1 & 1 \\
\hline
\end{tabular}

Most of the errors occurred in pronouncing the final sound of the word. The consonant sound $/ \mathrm{z} /, / \mathrm{d} /, / \theta /$, and the sound with silent pronunciation $/ \mathrm{m} /$ for $\mathrm{mb}$ in the thumb, and the $/ \mathrm{t} / \mathrm{at}$ Christmas appears to have much error in articulation.

The consonant sound /z/ was in the word zipper, house, pencils, Santa Claus, matches, pajamas, scissors. For the word zipper, it was pronounced in the initial sound. The respondents had no error with this since the sound corresponded to its letter; however, when the sound occurred in the word's final sound, the respondents committed much error in pronouncing it. The word ended with the letter $\mathrm{s}$, and the respondent pronounced it as /s/ instead of $/ \mathrm{z} /$. One reason was that respondents were not aware of the rules in pronouncing the word with final $-\mathrm{s}$ or -es. For the consonant sound /ð/, where the tip of the tongue should 
be positioned between the upper and lower teeth, respondents pronounced it in a manner where the tip of the tongue was pressed behind the upper front teeth producing the $/ \mathrm{d} / \mathrm{sound}$. This happened in pronouncing the $/ \theta /$ sound. Respondents articulate the sound as $/ t /$. For the word thumb and Christmas, the error was related to the pronunciation of silent letters. Respondents were not aware of when to pronounce a particular letter or the pronunciation rules when two consonants come together, especially when paired with nasalized consonants.

The error in the final consonant sound /z/ occurred because students did not have enough knowledge in phonological rules in pronouncing words with final $-\mathrm{s}$ or $-\mathrm{es}$. Thus language teachers should give focused on teaching phonological rules in pronunciation. It could also be implied that students do not have enough knowledge of the rules in pronouncing words ending with $-\mathrm{d}$ or $-\mathrm{ed}$. Moreover, rules in pronouncing the silent letters should be given attention. Language teachers should make the students realized why they need to be familiar with the rules

\section{CONCLUSION}

In light of the findings derived from the study, the following conclusions were drawn: The most significant percentage of consonant sounds mispronounced by the respondents with respect to the manner of articulation is fricative / $\mathrm{z} /$ followed by the fricative /ð/ and nasal $/ \mathrm{m} /$. As to the place of articulation, the most frequent error in pronouncing the consonant sounds of the respondents is on alveolar $/ \mathrm{z} /$, labiodental /ð/, and nasal $/ \mathrm{m} /$. English teachers have to teach the alveolar and labiodental fricatives in teaching the consonant sounds because most students failed to articulate these sounds correctly. Language teachers should also encourage students to engage themselves in practice exercises where their way of producing consonant sounds can be enhanced. It is also necessary to provide more reading materials and activities for soon to be teachers to enhance their consonant sound production. Provide training and development activities that would enhance Education students' pronunciation skills. These will help strengthen students' phonological awareness, especially that they will be teaching these sounds to their future students to avoid replicating the same errors. Due to this study's limitations, a thorough investigation of the same subject with a larger population is conducted to provide consistent results of the variables under investigation.

\section{REFERENCES}

Hassan, E. (2014 ). Pronunciation Problems: A Case Study of English Language Students at Sudan University of Science and Technology . English Language and Literature Studies; Vol. 4, No. 4, DOI:10.5539/ells.v4n4p31.

Bazzano, M. (2016). Deathlife, lifedeath. Reserachgate, DOI: 10.1080/14779757.2016.1204348.

Gray, C., Ferguson, J., Behan, S., Dunbar, C., Dunn, J., \& Mitchell, D. (2007). Developing young readers through the linguistic phonics approach. International Journal of Early Years Education, 15-33 DOI: 10.1080/09669760601106869. 
Knagg, J. (2019). How Many People Learn English? Retrieved from ThoughtCo.: retrieved from: https://www.thoughtco.com/how-many-people-learn-english-globally1210367\#: :text=There\%20are\%201.5\%20billion\%20English,British\%20Council $\% 20$ member\%20John\%20Knagg.

Lee, M.-L. (2009). "Differences in the Learning Anxieties Affecting College Freshman Students of EFL.". http://www.nus.edu.sg/celc/research/books/3rdsymposium/169to182-lee.pdf.

Nuhiu, M. (2012). Difficulties of Albanian speakers in pronouncing particular English speech sounds. Procedia- Social and Behavioral Sciences, 1703-1707.

Tayao, M. G. (2004, February). The evolving study of Philippine English phonology. Retrieved from World Englishes: https://onlinelibrary.wiley.com/doi/abs/10.1111/j.1467-971X.2004.00336.X

Wheelock, A. (2016). Phonological Difficulties Encountered by Italian Learners of English: An Error Analysis. Retrieved from https://www.hpu.edu/research-publications/tesolworking-papers/2016/04AnneWheelock.pdf. 